\title{
Analysis of Partial Discharge Features as Prognostic Indicators of Electrical Treeing
}

\author{
N. H. Ab Aziz ${ }^{1,2}$ and V. M. Catterson ${ }^{1}$ \\ ${ }^{1}$ Institute for Energy and Environment \\ University of Strathclyde \\ Glasgow, G1 1XW, United Kingdom \\ ${ }^{2}$ Faculty of Electrical Engineering \\ Universiti Teknikal Malaysia Melaka \\ Durian Tunggal, 76100 Melaka, Malaysia \\ S. M. Rowland ${ }^{3}$ \\ ${ }^{3}$ School of Electronic and Electrical Engineering \\ The University of Manchester \\ Manchester M13 9PL, United Kingdom \\ and S. Bahadoorsing ${ }^{4}$ \\ ${ }^{4}$ Department of Electrical and Computer Engineering \\ The University of the West Indies \\ St. Augustine, Trinidad and Tobago
}

\begin{abstract}
The aim of this paper is to identify promising indicators for prognosis of electrical treeing. Both phase-resolved partial discharge analysis (PRPDA) and pulse sequence analysis (PSA) are utilized. The prognostic properties of the features are evaluated in terms of monotonicity, prognosability, and trendability. The investigation reveals that PSA has a higher prognostic suitability index than PRPDA. An exponential fit is applied to the feature with the highest suitability index, in order to demonstrate its use for prognostic modeling and prediction of time until breakdown.
\end{abstract}

Index Terms - Electrical treeing, partial discharge, pulse sequence analysis, PRPD, prognostic, lifetime model, curve fitting.

\section{INTRODUCTION}

THE deterioration of insulation material in electrical power systems is a major concern for power utilities. The degradation process can lead to unplanned failures, with potentially lengthy downtime and associated costs. Such events can be reduced through condition-based asset management, with reliable prediction of the remaining useful life (RUL) of assets. Based on current health information, a prognostic system which accurately predicts RUL could avoid unscheduled downtimes, thus reducing costs for repair and replacement.

Partial discharge (PD) analysis has been widely used in insulation failure diagnosis. The most common approach has traditionally been through the phase-resolved partial discharge (PRPD) representation, until pulse sequence analysis (PSA) became an alternative in the 1990's [1]. In the PRPD, the

Manuscript received in final form 11th August 2016. variation of PD pulses (either in magnitude or repetition rate) is often represented by statistical quantities. Early examples of this approach can be found in $[2,3]$. Later, an automated approach to knowledge-based PD recognition was introduced in $[4,5]$ that mimics the approach of PD experts in [6-8] for PD interpretation.

The most common method of PRPD analysis (PRPDA) is to extract 2-dimensional (2D) distributions from the 3D $\phi-q-n$ plot e.g. discharge pulse count distribution, $H_{n}(\phi)$, discharge maximum pulse height distribution, $H_{q m}(\phi)$, and discharge average pulse height distribution, $H_{q n}(\phi)$. The description and characteristics of the PD distributions are often represented by statistical quantities e.g mean, variance, skewness and kurtosis [2-5, 9-11]. However, the analysis of accumulated discharge data in PRPDA gives inadequate information on the fundamental physical phenomena for the interpretation of the degradation process [1]. 
The analysis of discharge sequences is suggested in PSA as an alternative with a more meaningful interpretation of PD phenomena. The strong correlations that exist between consecutive discharge pulses are influenced by space charge and the polarity of the instantaneous voltage [12]. Thus, the key parameter of PSA is the change of electric field between consecutive pulses which is reflected in the change in voltage due to the excitation waveform. In some cases, time intervals between consecutive discharges give a distinctive pattern. PSA has been applied for diagnosis in [12-16] hence should be considered for prognosis. The voltage change, $\Delta U$ and the ratio of $\Delta U / \Delta t$ are typical parameters used for this approach, either for PD pattern recognition [12-14] or for characterizing the degradation stages $[15,16]$.

Electrical trees progress by periodic PDs after initiating at a point of high divergent stress [17]. Hence, PD measurement has long been associated with electrical treeing for detection [18], determining the type of electrical tree (e.g. branch or bush) [19] and classifying the growth stage [20]. However, prognosis is a further stage beyond diagnosis with the aim of predicting when failure will occur. In prognosis, it is important to select appropriate degradation parameters for RUL or timeto-failure (TTF) estimation.

A set of metrics was introduced in [21] which characterize the suitability of a prognostic parameter. Three main qualities of an ideal prognostic parameter have been proposed: monotonicity, prognosability, and trendability. Monotonicity characterizes the trend of time series data considering how consecutive data behaves i.e increasing, decreasing, or static. A parameter with high monotonicity will have a strong trend in one direction, and will consequently provide good information for a prognostic model. Prognosability indicates the distribution of failure values in relation to the initial values of a population of systems, i.e. the repeatability of the specific failure value between different samples. Samples that fail when a parameter reaches a particular threshold value (with a narrow distribution) will have high prognosability, and it will therefore be easier to predict the point of failure using this parameter than another with lower prognosability. Finally, trendability gives the smallest correlation coefficient between two samples in a population, which indicates the level of similarity of the shape of the failure curve between two samples. A parameter which consistently corresponds to one failure curve will be easier to model than another with low trendability.

This paper demonstrates the advantage of PSA fingerprints over PRPDA as prognostic indicators for insulation lifetime prediction. Large numbers of features can be extracted from both PRPDA and PSA approaches, hence, a further stage is required to reduce the dimensionality of the features (feature transformation) or to pick only the significant ones (feature selection). A common technique for the former is principle component analysis (PCA) [22] although linear discriminant analysis (LDA) has been claimed to give a better transformation in [23]. Genetic optimization [24] and particle swarm optimization [25] are examples of the latter approach.
In this paper however, the best feature is selected through the three prognostic metrics described previously.

The summary of the methodology for identifying the best prognostic indicator is outlined in Section 2. Section 3 describes the preparation of PD data and the following section explains how the features from PRPDA and PSA are extracted. The best feature is identified in Section 5 and a proposed prognostic model is presented in Section 6.

\section{METHODOLOGY}

The aim of this research work is to predict the lifetime of insulation samples due to electrical treeing. To achieve this goal, it is vital to identify the best feature or combination of features that track with the degradation process. In this paper, PD measurements from electrical treeing experiments reported in [26] are employed as the degradation data. Epoxy resin is used as the dielectric in point-plane test samples, allowing observation and measurement of the tree growth. Throughout the tree growth, PD data is recorded in 5-minute batches. A brief description on the data collection can be found in Section 3 .

A total of 21 samples are selected and analyzed using PRPDA and PSA approaches in order to identify the prognostic features as described in Section 4. As a result, 24 features are extracted from PRPDA and 7 from PSA. Since the features can be considered inter-correlated, feature selection is employed rather than feature transformation. The selection is based on the three prognostic metrics, described in Section 5.

Finally, a prognostic model is proposed using exponential fitting and a thorough discussion in Section 6 aids the calculation of important properties of the model for insulation lifetime prediction.

\section{DATA COLLECTION}

The PD data from electrical treeing experiments reported in [26] is employed in this study. The main purpose of the prior experiments was to investigate the role of power quality on electrical treeing in epoxy resin, thus these samples were subjected to various harmonic regimes. The experiment utilized a total of seven different harmonicinfluenced test waveforms including the fundamental (named Wave 1, 7, 8, 9, 11, 12 and 13) as described in [27], and with equations as indicated in (1) to (7) below. The effects of the harmonics on the PSA plots have been investigated in [28], which revealed some differences for waveforms with high total harmonic distortion (THD). In this work however, the differences are less significant as data is aggregated into features representing 5 minute batches. As observed in [27], at this level of aggregation there is no obvious pattern for discriminating the harmonic groups.

$$
\begin{aligned}
& u_{1}(t)=10.25 \sqrt{2}(\sin \theta+0.4 \sin 3 \theta) \\
& u_{7}(t)=10.18 \sqrt{2}(\sin \theta) \\
& u_{8}(t)=9.7 \sqrt{2}(\sin \theta+0.05 \sin 5 \theta)
\end{aligned}
$$




$$
\begin{aligned}
& u_{9}(t)=10.4 \sqrt{2}(\sin \theta+0.05 \sin 7 \theta) \\
& u_{11}(t)=9.35 \sqrt{2}(\sin \theta+0.178 \sin 7 \theta) \\
& u_{12}(t)=10.15 \sqrt{2}(\sin \theta+0.032(\sin 5 \theta+ \\
& \sin 7 \theta+\sin 11 \theta+\sin 13 \theta+\sin 23 \theta+\sin 25 \theta)) \\
& u_{13}(t)=10.18 \sqrt{2}(\sin \theta+0.02(\sin 5 \theta+\sin 7 \theta+ \\
& \sin 11 \theta+\sin 13 \theta+\sin 23 \theta+\sin 25 \theta))
\end{aligned}
$$

Electrical treeing was induced through a point-plane arrangement of hypodermic needle (tip radius $=3 \mu \mathrm{m}$, length $=$ $51 \mathrm{~mm}$ and thickness $=1.1 \mathrm{~mm}$ ) in epoxy resin encased in an acrylic cube $(25 \mathrm{~mm} \times 25 \mathrm{~mm} \times 25 \mathrm{~mm})$ with a plane separation gap of $2 \pm 0.5 \mathrm{~mm}$. The testing facilities and preparation of the samples are described in detail in [29] including the removal process of the lubricant coating from the needle.

The detail of the experimental plan can be found in [26] and is summarized here. The first stage is to initiate electrical treeing under the influence of $50 \mathrm{~Hz}$ at $18.0 \mathrm{kV}$ peak within 30 minutes. After that, the composite waveform was increased to $14.4 \mathrm{kV}$ peak and maintained for a maximum of 2.5 hours while PD data consisting of phase and discharge magnitude was recorded continuously in 5 minute batches until breakdown occurred. Out of 42, only 31 samples reached breakdown within 2.5 hours. Of these, 28 samples were used in this study, due to very low scores of the monotonicity and trendability metrics from the remaining three. 21 tree samples were utilized for model training, with the remaining seven used for model validation.

\section{PD FEATURES}

In this section, the changes of $\mathrm{PD}$ properties from PRPDA and PSA are observed throughout the tree growth in order to identify possible features. The behavior of each feature is ranked with prognostic metrics for feature reduction that will be discussed in the next section.

\subsection{PRPDA}

Figure 1 shows the PRPD pattern of a sample tested with Wave 1 at the early and late stages of growth. During the early growth, we can see that the positive pulses are concentrated along the rising slope of the sine wave and the negative pulses along the falling slope. However, both positive and negative pulses appear along the rising and falling slopes during the late growth. No PD activity was recorded after the peaks where the slope, $d v / d \theta$ is zero. The same pattern holds for Wave 7, 8, 9, 11, 12 and 13. Prior to breakdown, three changes can be spotted in this figure (based on a 5-minute batch of data) which seem correlated to the tree growth:

i. The number of PD pulses increases

ii. The maximum PD magnitude increases

iii. The phase distribution widens

In order to extract these changes from the PRPD pattern, the $3 \mathrm{D}$ plots of $\phi-q-n$ were transformed into the $2 \mathrm{D}$ plots of number of discharges $H_{n}(\phi)$, maximum values of discharges $H_{\text {qmax }}(\phi)$, and average values of discharges $H_{q n}(\phi)$, as in [8]. To generate these three plots, the $360^{\circ}$ voltage cycle is divided into 60 equal-width buckets. For each bucket, the number of discharges, $n$, the maximum value of discharge, $q_{\max }$, and the average value of discharge, $q_{n}$, are determined using absolute charge values. These values are then plotted to produce the three $2 \mathrm{D}$ phase distribution plots as shown in Figure 2 (in reference to Figure 1). Among the three 2D distributions, $H_{n}(\phi)$ gives the most similar envelope to the $\phi-q$ - $n$ plots.

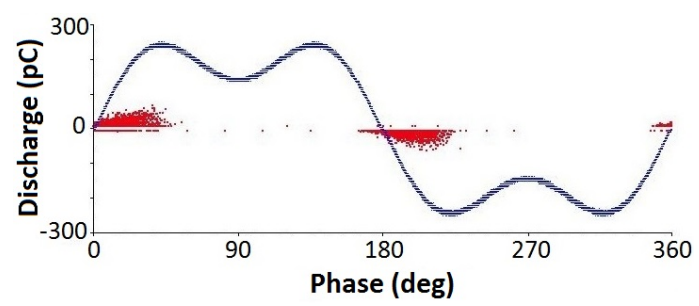

(a) Early growth

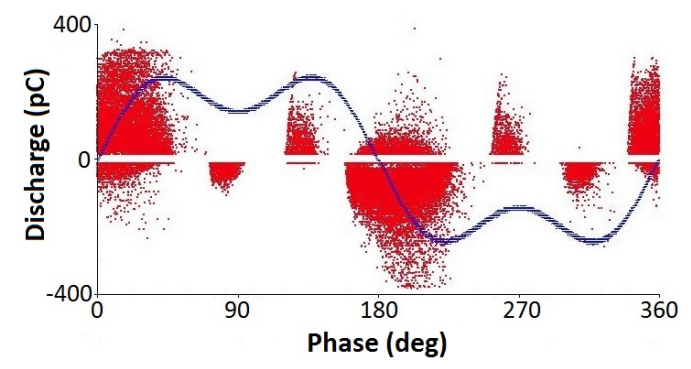

(b) Late growth

Figure 1. PRPD pattern of a sample tested with Wave 1

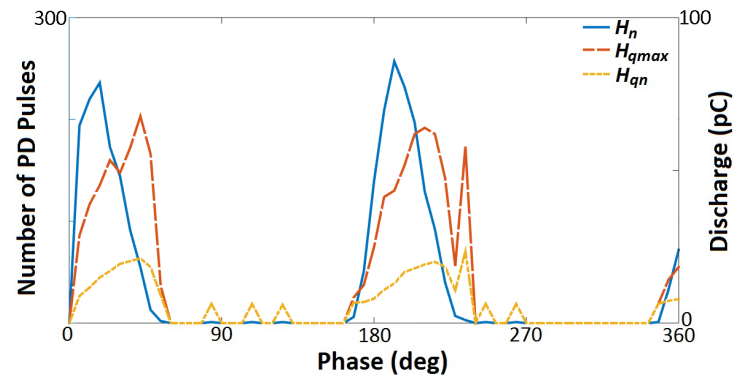

(a) Early growth

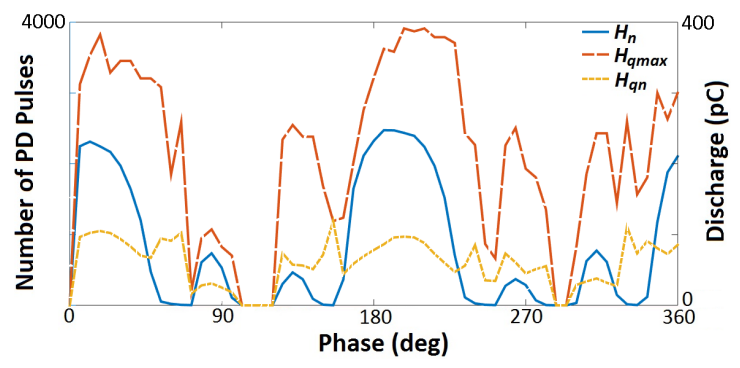

(a) Late growth

Figure 2. $H_{n}(\phi), H_{q \max }(\phi)$ and $H_{q n}(\phi)$ distributions of Figure 1

The distribution of the 2D pattern is represented by four statistical moments, i.e mean, $\mu$, standard deviation, std, skewness, $s k$, and kurtosis, $k u$. These four moments are calculated for the positive and negative half cycles of the 
three 2D plots mentioned previously, giving a total of 24 features.

The list of statistical features to be selected as prognostic indicators for each 2D PRPD pattern is tabulated in Table 1 (feature no. 1-24) with a total of 24 features from PRPDA. All these features are calculated from 5 minute batches of data. From observation, the negative and positive half cycles of each statistical feature shows a similar trend. Thus, only positive features are showed here for discussion.

Among the four statistical features, the mean and standard deviation for the three $H_{n}(\phi), H_{q \max }(\phi)$, and $H_{q n}(\phi)$ distributions show an increasing trend compared with skewness and kurtosis. For illustration, the mean, standard deviation, skewness, and kurtosis of the $H_{n}(\phi)$ distribution of all 21 samples are shown in Figure 3 since $H_{n}(\phi)$ shows the clearest trend. The plots show each feature against timeto-failure (TTF) as denoted in equation (8). The red * indicates the failure values which will be referred in Section 5. The mean and standard deviation show an increasing pattern throughout the tree growth although with a sudden decrease around 50 minutes before breakdown. The skewness and kurtosis however, show a very slightly decreasing pattern because of their smaller range compared to mean and standard deviation.

$$
T T F=t-t_{b d}
$$

where TTF is the time-to-failure, $t$ is the experimental time when the data is taken and $t_{b d}$ is the breakdown time of the sample.

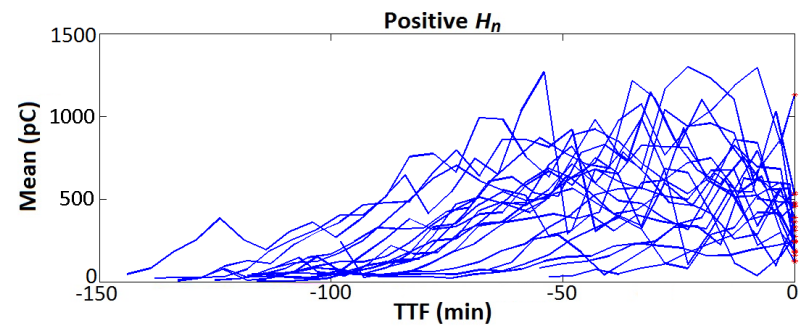

(a) Mean of positive $H_{n}$

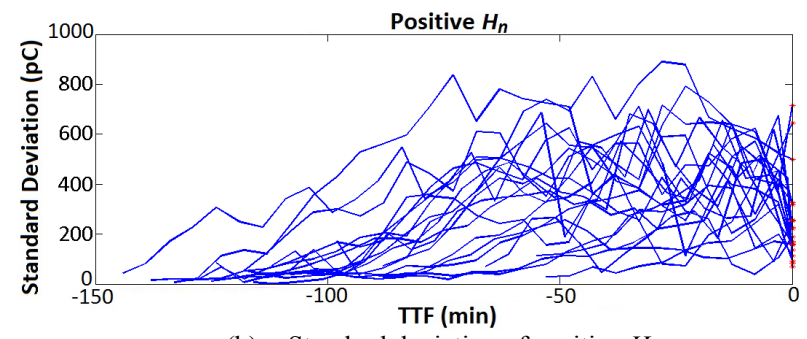

(b) Standard deviation of positive $H_{n}$

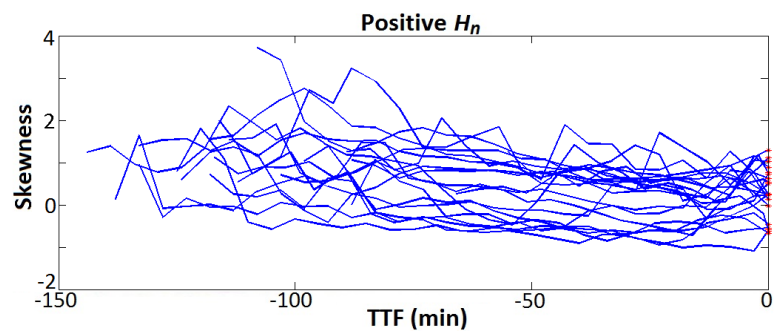

(c) Skewness of positive $H_{n}$

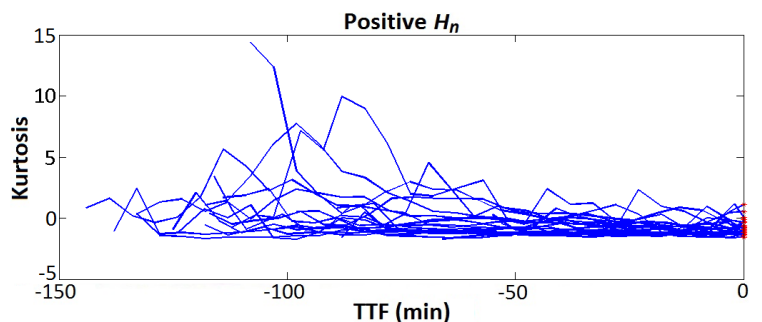

(d) Kurtosis of positive $H_{n}$

Figure 3. Statistical analysis of positive $H_{n}$ distribution, (a) mean, (b) standard deviation, (c) skewness and (d) kurtosis

\subsection{PSA}

The most common parameter used for PSA is the voltage change between the consecutive pulses [12-14, 16]. In order to determine the changes, the instantaneous voltage, $u(t)$, of every PD pulse is calculated using phase position, $\theta$, and one of equations (1) to (7) for test waveforms 1, 7, 8, 9, 11, 12 and 13 as appropriate. $\Delta u\left(t_{i}\right)$ is then determined using equation (9) as illustrated in Figure 4 where $i$ is the current current PD pulse and $i-1$ is the previous one.

$$
\Delta u\left(t_{i}\right)=u\left(t_{i}\right)-u\left(t_{i-1}\right)
$$

Referring to Figure 4, taking sample from Wave 7 as an example, we can see that more PD pulses occurred during the late growth, thus decreasing the $\Delta u$ and $\Delta \theta$. As these key changes can be considered to be linked by the slope between the consecutive pulses, in this study three parameters are considered for further investigation, $\Delta u, \Delta \theta$ and $\Delta u / \Delta \theta$.

The PD pulses in Figure 4 can be divided into four clusters as shown in the legend. Clusters $\mathrm{A}$ and $\mathrm{C}$ are $\mathrm{PD}$ pulses in the rising and falling parts of the voltage waveform respectively where the $\Delta u$ and $\Delta \theta$ are considered small. The PD pulses at the peaks and just after the peaks form $\mathrm{B}$ and $\mathrm{D}$ clusters that have greater $\Delta u$ and $\Delta \theta$. Therefore, features $\Delta u$ and $\Delta \theta$ can be divided into two groups: small (voltage difference between consecutive pulses in Clusters A and C) and large (voltage difference between consecutive pulses in Clusters B and D). 


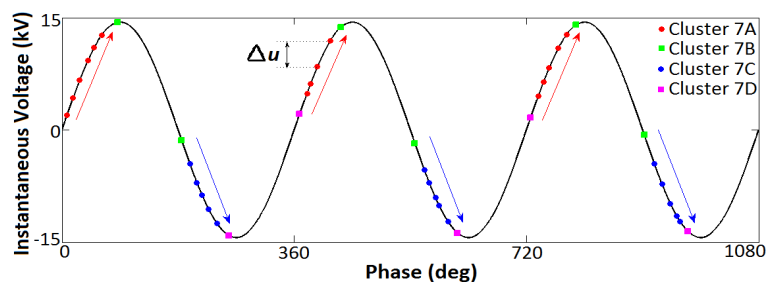

(a) Early growth

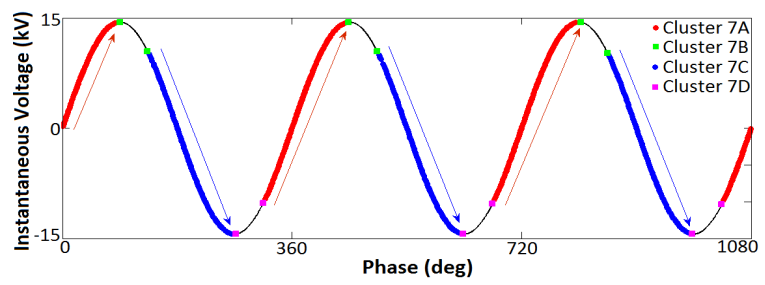

(b) Late growth

Figure 4. The instantaneous voltage, $u(t)$, of PD occurrence overlaid on Wave 7

The $\Delta u$ and $\Delta \theta$ features were calculated from the small group only, from the large group only, and from all PD pulses ignoring which group they belong to. However, the $\Delta u / \Delta \theta$ behaves differently and thus was calculated from all data only. For a 5 minute batch of data, the average value of each feature is determined. In total, 7 potential features have been extracted from PSA as tabulated in Table 1 (feature no. 25-31). For illustration purposes, 3 PSA features are shown in Figure 5: voltage difference of all PD pulses, $\Delta u_{\text {all }}$, phase difference of all PD pulses, $\Delta \theta_{\text {all }}$, and derivative of voltage, $\Delta u / \Delta \theta$, indicating how the features change towards breakdown.

Interestingly, $\Delta u_{\text {all }}$ and $\Delta \theta_{\text {all }}$ show a very similar pattern of decreasing exponentially throughout the tree growth. Voltage derivative however shows a different pattern with an increase during the early growth which decreases slowly towards the breakdown. Also, it appears visually that PSA features show a smoother pattern compared to PRPDA in Figure 3 .

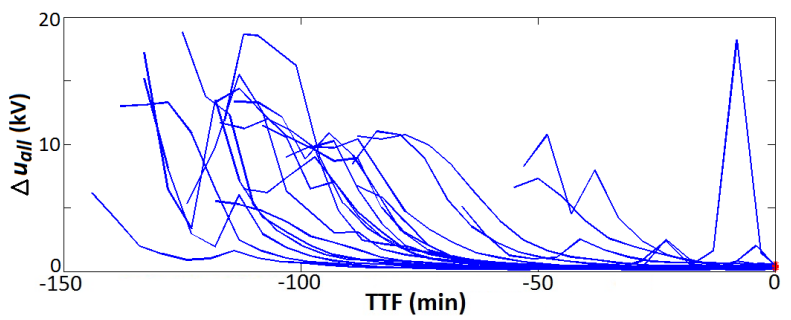

(a) Voltage difference of all PD pulses, $\Delta u_{\text {all }}$

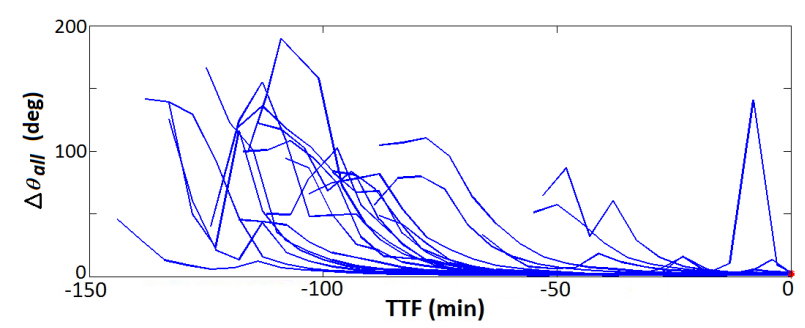

(b) Phase difference of all PD pulses, $\Delta \theta_{\text {all }}$

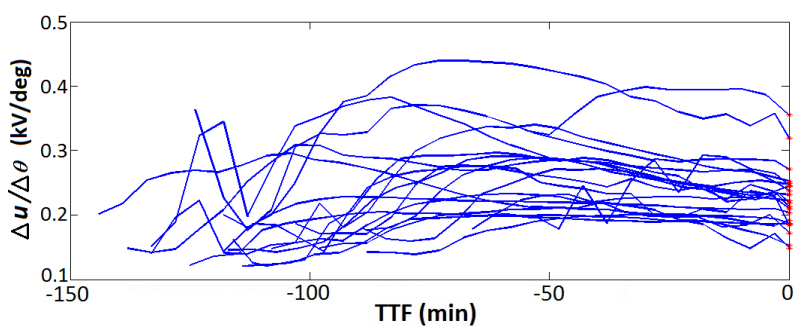

(c) Derivative of voltage, $\Delta u / \Delta \theta$

Figure 5. Three of the PSA features

\section{FEATURE REDUCTION}

Results from the previous section reveal 24 features from PRPDA and 7 features from PSA. The next stage is to reduce the number of features and to consider only the significant ones for lifetime model development. The total of the three metrics in [21], i.e monotonicity, prognosability, and trendability as indicated in equations (10)-(12) respectively, is considered for feature reduction. The total is given by equation (14).

$$
\begin{gathered}
\text { Monotonicity }=\text { mean }\left|\frac{\# \frac{d}{d x}>0}{N-1}-\frac{\# \frac{d}{d x}<0}{N-1}\right| \\
\text { Prognosability }=\exp \left[-\frac{\operatorname{std}\left(p_{\text {fail }}\right)}{\operatorname{mean}\left|p_{\text {start }}-p_{\text {fail }}\right|}\right] \\
\text { trendability }=\min \left(\left|\operatorname{corrcoe} f_{x y}\right|\right) \\
\text { corrcoef } f_{x y}=\frac{n\left(\sum x y\right)-\left(\sum x\right)\left(\sum y\right)}{\sqrt{\left[n \sum x^{2}-\left(\sum x\right)^{2}\right]\left[n \sum y^{2}-\left(\sum y\right)^{2}\right]}} \\
\text { Total }=\text { Monotonicity }+ \text { Prognosability }+ \text { Trendability }
\end{gathered}
$$

where $\# \frac{d}{d x}>0$ is the number of sequential datapoints where the second is greater than the first (positive $\frac{d}{d x}$ ), $\# \frac{d}{d x}<0 \quad$ is the number of sequential datapoints where the second is less than the first (negative $\frac{d}{d x}$ ), $N$ is the number of observations, std is the standard deviation and $p$ is the value of the prognostic feature.

Monotonicity is an important feature of a prognostic indicator as the degradation parameters change when the system degrades. Based on equation (10), the monotonicity score will be higher if most of the feature values change in one direction. This can be seen in PSA features in Figure 5 that are less fluctuated compared to PRPDA features in Figure 3 thus results in higher score of monotonicity.

Referring to equation (11), prognosability will be higher if the failure values (indicated by red * in Figure 3 and 5) have small standard deviation and the range of the parameter is large. This can be observed in Figure 3 where skewness and kurtosis have a smaller distribution of the failure values compared to mean and standard deviation giving a higher score of prognosability for the former (feature no. 3 and 4 in Table 1 have a higher score compared to no. 1 and 2). However, the skewness and kurtosis in Figure 3 have lower scores than $\Delta u_{\text {all }}$ and $\Delta \theta_{\text {all }}$ in Figure $5 \mathrm{a}$ and $5 \mathrm{~b}$ respectively due to the smaller parameter range of the former. 
For trendability, the quantile-quantile ( $\mathrm{q}-\mathrm{q})$ plot is performed for every sample combination due to the different sample sizes. This is to determine whether two data sets have a common distribution. The linearity of the relationship is measured using Pearson's correlation coefficient in equation (13). The final value is given by the smallest absolute correlation as indicated in equation (12).

All the features in Table 1 are then ranked based on the sum of the prognostic metrics as in equation (14). Each of these metrics ranges from zero to one, one indicating a very high score on that metric and zero indicating that the parameter is not suitable according to that metric. Overall, PSA features have better scores for all three prognostic metrics and the total suitability compared to PRPDA. The great difference between those two approaches can be seen in trendability scores showing less consistent behavior among PRPDA features. Generally, features with an overall score greater than 2 should be considered as reasonable prognostic indicators. Based on overall score, $\Delta u_{\text {all }}$ is chosen as the prognostic indicator for the life time model, described in the next section.

TABLE 1. Prognostic metrics of PRPDA and PSA features, where mono is monotonicity, prog is prognosability and trend is trendability

\begin{tabular}{ccccccc}
\hline No & Features & Mono & Prog & Trend & Total & Rank \\
\hline $\mathbf{1}$ & $H_{n}$ pos $\mu$ & 0.28 & 0.48 & 0.0101 & 0.78 & 15 \\
$\mathbf{2}$ & $H_{n}$ pos std & 0.27 & 0.42 & 0.0070 & 0.69 & 17 \\
$\mathbf{3}$ & $H_{n}$ pos $s k$ & 0.19 & 0.70 & 0.004 & 0.89 & 13 \\
$\mathbf{4}$ & $H_{n}$ pos $k u$ & 0.18 & 0.78 & 0.0026 & 0.96 & 9 \\
$\mathbf{5}$ & $H_{n}$ neg $\mu$ & 0.34 & 0.59 & 0.0431 & 0.96 & 9 \\
$\mathbf{6}$ & $H_{n}$ neg std & 0.32 & 0.46 & 0.0052 & 0.79 & 14 \\
$\mathbf{7}$ & $H_{n}$ neg sk & 0.25 & 0.75 & 0.0209 & 1.02 & 7 \\
$\mathbf{8}$ & $H_{n}$ neg $k u$ & 0.18 & 0.82 & 0.0022 & 1.00 & 8 \\
$\mathbf{9}$ & $H_{\text {qmax }}$ pos $\mu$ & 0.30 & 0.62 & 0.0053 & 0.93 & 11 \\
$\mathbf{1 0}$ & $H_{\text {qmax }}$ pos std & 0.16 & 0.53 & 0.0014 & 0.69 & 17 \\
$\mathbf{1 1}$ & $H_{\text {qmax }}$ pos $s k$ & 0.12 & 0.39 & 0.0004 & 0.52 & 27 \\
$\mathbf{1 2}$ & $H_{\text {qmax }}$ pos $k u$ & 0.12 & 0.11 & 0.0021 & 0.24 & 31 \\
$\mathbf{1 3}$ & $H_{\text {qmax }}$ neg $\mu$ & 0.29 & 0.63 & 0.0036 & 0.92 & 12 \\
$\mathbf{1 4}$ & $H_{\text {qmax }}$ neg std & 0.20 & 0.54 & 0.0125 & 0.75 & 16 \\
$\mathbf{1 5}$ & $H_{\text {qmax }}$ neg $s k$ & 0.13 & 0.48 & 0.0027 & 0.61 & 21 \\
$\mathbf{1 6}$ & $H_{\text {qmax }}$ neg $k u$ & 0.13 & 0.20 & 0.0052 & 0.33 & 29 \\
$\mathbf{1 7}$ & $H_{\text {qn }}$ pos $\mu$ & 0.20 & 0.41 & 0.0016 & 0.61 & 21 \\
$\mathbf{1 8}$ & $H_{\text {qn }}$ pos std & 0.16 & 0.39 & 0.0008 & 0.55 & 25 \\
$\mathbf{1 9}$ & $H_{\text {qn }}$ pos $s k$ & 0.09 & 0.53 & 0.0042 & 0.62 & 20 \\
$\mathbf{2 0}$ & $H_{\text {qn }}$ pos $k u$ & 0.11 & 0.42 & 0.0034 & 0.53 & 26 \\
$\mathbf{2 1}$ & $H_{\text {qn }}$ neg $\mu$ & 0.23 & 0.38 & 0.0021 & 0.61 & 21 \\
$\mathbf{2 2}$ & $H_{\text {qn }}$ neg std & 0.12 & 0.40 & 0.0013 & 0.52 & 27 \\
$\mathbf{2 3}$ & $H_{\text {qn }}$ neg sk & 0.14 & 0.51 & 0.0040 & 0.66 & 19 \\
$\mathbf{2 4}$ & $H_{\text {qn }}$ neg $k u$ & 0.11 & 0.16 & 0.0035 & 0.28 & 30 \\
$\mathbf{2 5}$ & $\Delta \boldsymbol{u}_{\text {all }}$ & $\mathbf{0 . 6 5}$ & $\mathbf{0 . 9 9}$ & $\mathbf{0 . 4 8 9 8}$ & $\mathbf{2 . 1 2}$ & $\mathbf{1}$ \\
$\mathbf{2 6}$ & $\Delta u_{\text {small }}$ & 0.61 & 0.98 & 0.3871 & 1.98 & 5 \\
$\mathbf{2 7}$ & $\Delta u_{\text {large }}$ & 0.59 & 0.77 & 0.2453 & 1.60 & 6 \\
$\mathbf{2 8}$ & $\Delta \theta_{\text {all }}$ & 0.63 & 0.99 & 0.4576 & 2.08 & 2 \\
$\mathbf{2 9}$ & $\Delta \theta_{\text {small }}$ & 0.60 & 0.98 & 0.4250 & 2.01 & 4 \\
$\mathbf{3 0}$ & $\Delta \theta_{\text {large }}$ & 0.70 & 0.83 & 0.5365 & 2.06 & 3 \\
\hline & & & & & &
\end{tabular}

\begin{tabular}{lllllll}
\hline 31 & $\Delta u / \Delta \theta$ & 0.17 & 0.38 & 0.0085 & 0.56 & 24 \\
\hline
\end{tabular}

\section{PROGNOSTIC MODELING}

The main focus of this paper is the methodology for identifying an appropriate prognostic feature, and the result that $\Delta u_{\text {all }}$ is best for electrical treeing according to the prognostic metrics. However, this choice of feature must be used within a prognostic model in order to demonstrate its capabilities. This section introduces a relatively simple prognostic model which utilizes the $\Delta u_{\text {all }}$ feature.

A comprehensive review in [30] has discussed the development of insulation life models. The author pointed out that the prognostic study of polymeric insulation in the last two decades has been focused on accelerated life tests including thermal, electrical, and thermo-electrical endurance which often fit either the exponential (global degradation mechanism) or inverse-power model (local defects e.g. PD initiation and treeing). The parameters of these so-called phenomenological life models were determined from the relationships between mean-time-to-failure (MTTF) and applied stresses with no physical knowledge involved. Thus in this study, both fitting curves are considered and the average of the goodness of fit (GOF) for all 21 training samples are tabulated in Table 2. Based on the GOF, exponential curve fitting is applied for prognostic modeling.

TABLE 2. Goodness of fit (GOF), where $S S E$ is the sum of squared error and RMSE is the root mean squared error.

\begin{tabular}{cccc}
\hline $\begin{array}{c}\text { Curve } \\
\text { Fitting }\end{array}$ & SSE & $\begin{array}{c}\text { Adjusted } \\
\text { R-Square }\end{array}$ & RMSE \\
\hline Exponential & 54.97 & 0.84 & 1.33 \\
Inverse-power & 111.70 & 0.69 & 2.02 \\
\hline
\end{tabular}

Figure 6 shows the best feature selected in Section 5, $\Delta u_{\text {all }}$, of a sample tested with Wave 11, plotted towards breakdown. From the plot, 3 stages can be seen. Stage 1 normally occurs for 10 to 20 minutes after the tree initiated where the feature does not closely fit the curve. At this stage, the electrical tree was mainly growing towards the ground plate. In Stage 2, the tree starts to grow rapidly either in length (greater tree length per min) or width (more tree branches) and then slows at the end of the stage. During the rapid growth, a great difference in $\Delta u_{\text {all }}$ can be seen in the plot yielding a steeper slope. Finally, Stage 3 is the longest stage with fluctuation and very slow growth rate. At this stage the voltage difference between consecutive pulses does not vary much even although this is the stage where breakdown occurs, therefore the $\Delta u_{\text {all }}$ feature is quite constant in this region. One of the reasons for the small $\Delta u_{\text {all }}$ is because of the increasing number of PD pulses in this region which decreases the time difference between the consecutive PDs thus decreasing the $\Delta u_{\text {all }}$. In order to clearly view points in Stage 3, a log scale is applied to the $\Delta u_{\text {all }}$ as shown in Figure 7. 


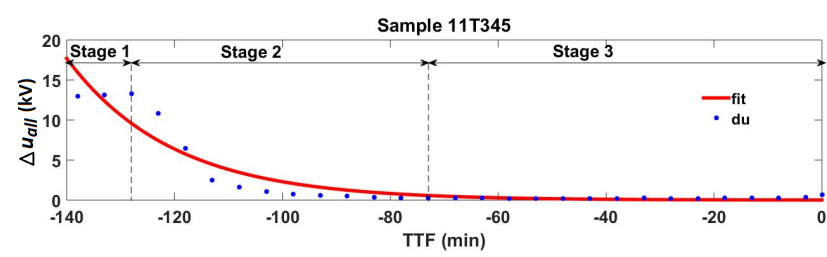

Figure 6. $\Delta u_{\text {all }}$ of sample $11 \mathrm{~T} 345$

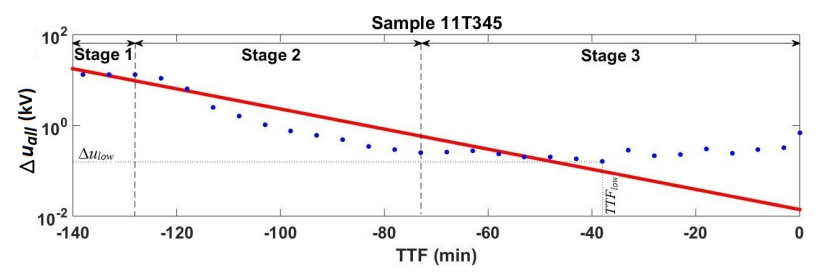

Figure 7. Exponential fit of sample 11T345 in semilog scale

The exponential fitting in Figure 6 and 7 considers all data points of sample 11T345. The exponential does not always closely fit the data points because of relatively constant $\Delta u_{\text {all }}$ values in Stages 1 and 3. Those Stage 1 values however, produce a fit with lower slope that passes through the lowest $\Delta u_{\text {all }}$ value, $\Delta u_{\text {low }}$, which was at 38 minutes TTF. $\Delta u_{\text {all }}$ after this point deviates from the exponential curve. Most of the training samples show the same behavior. For this reason, the prognostic model is used to predict the time when the fitted curve crosses the threshold of $\log$ of $\Delta u_{\text {low }}, t_{\text {low }}$, rather than the time of breakdown. For validation, the estimated $t_{\text {low }}, t_{\text {low(est) }}$, is determined using the rule-based approach with the knowledge of the slope of the semilog exponential fit, $m_{\text {low }}$ and $t_{\text {low }}$ (from exponential fit).

For each of the training samples, the values of $\Delta u_{\text {low }}, t_{\text {low }}$, $m_{\text {low }}$, and the TTF after $t_{\text {low }}$ were calculated. Summary statistics are shown in Table 3. It can be seen that the mean $\log \Delta u_{\text {low }}$ is -0.69 , and the average TTF after $t_{\text {low }}$ is 19.3 minutes. Therefore, the prognostic model is applied to new sample data as follows:
i. Perform the exponential fit
ii. Determine $t_{\text {low }}$ when the $\log \Delta u_{\text {low }}$ reaches -0.69
iii. Predict the breakdown time, $t_{b d}$, using equation (15)

Table 3 lists the slope of the semilog exponential fit at time $t_{\text {low }}, m_{\text {low }}$. It can be seen that there is a very small standard deviation of $m_{\text {low }}$, meaning that all samples have a very similar rate of change in $\Delta u_{\text {all }}$ at this point. Therefore, while there is a relatively large standard deviation for the TTF characteristic, $m_{\text {low }}$ suggests that the model is capturing underlying similar behavior between the samples. This makes $m_{\text {low }}$ as the most important properties in the rulebased approach for determining $t_{\text {low(est) }}$.

TABLE 3. Properties of prognostic model of $\Delta u_{\text {all }}$

\begin{tabular}{cccccc}
\hline Properties & Min & Max & Range & Mean & $\begin{array}{c}\text { Standard } \\
\text { deviation }\end{array}$ \\
\hline Log $\Delta \boldsymbol{u}_{\text {low }}$ & -0.87 & -0.35 & 0.52 & -0.69 & 0.14 \\
$\boldsymbol{T} \boldsymbol{T} \boldsymbol{F}_{\text {low }}$ & $0 \mathrm{~min}$ & $54 \mathrm{~min}$ & $54 \mathrm{~min}$ & $19 \mathrm{~min}$ & $16 \mathrm{~min}$ \\
\hline
\end{tabular}

\begin{tabular}{cccccc}
\hline $\boldsymbol{t}_{\text {low }}$ & $58 \mathrm{~min}$ & $114 \mathrm{~min}$ & $56 \mathrm{~min}$ & $92 \mathrm{~min}$ & $16 \mathrm{~min}$ \\
$\boldsymbol{m}_{\text {low }}$ & $|-0.017|$ & $|-0.025|$ & 0.008 & -0.02 & -0.002 \\
\hline
\end{tabular}

$$
t_{b d}=t_{\text {low }(e s t)}+19 \min
$$

As a final test, the same seven samples as in [27] were used to validate the $\Delta u_{\text {all }}$ model over time. Figure 8 shows the predicted TTF of a sample tested with Wave 1 (actual breakdown time is 115 minutes). During early time instances, the behavior of $\Delta u_{\text {all }}$ in Stage 1 results in a relatively large variance in the TTF estimation. After entering Stage 2, the error becomes smaller, and the accurate TTF is predicted consistently after 53 minutes.

The remaining six samples show broadly similar behavior, with accuracy of prediction increasing during Stage 2 of tree growth. The average time at which the predictions converge with the true value for each of the test samples is 51 minutes. The average of the time difference between the actual and predicted TTF decreases from 13 minutes before the convergence to 0.3 minutes after the convergence. This behavior may be expected, as the accuracy of the exponential fit should increase with the amount of data.

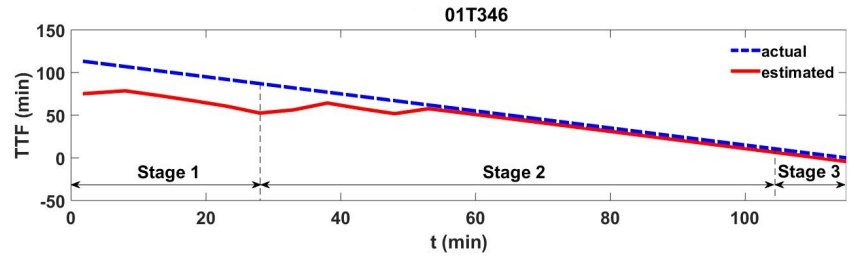

Figure 8. Actual and estimated TTF of a test sample

\section{CONCLUSIONS AND FUTURE WORK}

This paper utilized PRPDA and PSA approaches in identifying possible prognostic indicators of breakdown due to electrical treeing. Between these two approaches, features from PSA have greater prognostic suitability, based on the total of the three vital metrics of monotonicity, prognosability, and trendability. Feature $\Delta u_{\text {all }}$ has the highest suitability using this metric and was chosen as the prognostic indicator for lifetime modeling employing exponential fitting. Results show that accuracy of prediction of time to breakdown increases as more data becomes available for exponential fitting, in particular after tree growth enters Stage 2.

The prognostic modeling approach of curve fitting was taken here due to the relative simplicity of the technique, and its capabilities for visualizing the predictions over time (Figure 6 and 7). Future work will investigate alternative approaches to prognostic modeling utilizing the same feature, by following a prognostic design methodology such as that reported in [31].

\section{REFERENCES}


[1] M. Hoof and R. Patsch, "Analyzing partial discharge pulse sequences-A new approach to investigate degradation phenomena," in Conference Record of the 1994 IEEE International Symposium on Electrical Insulation, 1994, pp. 327-331.

[2] T. Okamoto and T. Tanaka, "Novel partial discharge measurement computer-aided measuremnet systems," IEEE Transactions on Electrical Insulation, no. 6, pp. 1015-1019, 1986.

[3] E. Gulski and F. Kreuger, "Computer-aided analysis of discharge patterns," Journal of Physics D: Applied Physics, vol. 23, no. 12, p. 1569, 1990.

[4] S. Strachan, S. Rudd, S. McArthur, M. Judd, S. Meijer, and E. Gulski, "Knowledge-based diagnosis of partial discharges in power transformers," IEEE Transactions on Dielectrics and Electrical Insulation, vol. 15, no. 1, pp. 259-268, 2008.

[5] S. Rudd, S. McArthur, and M. Judd, "A generic knowledge-based approach to the analysis of partial discharge data," IEEE Transactions on Dielectrics and Electrical Insulation, vol. 17, no. 1, pp. 149-156, 2010.

[6] G. P. Cleary and M. D. Judd, "UHF and current pulse measurements of partial discharge activity in mineral oil," IEE ProceedingsScience, Measurement and Technology, vol. 153, no. 2, pp. 47-54, 2006.

[7] M. D. Judd, L. Yang, and I. B. Hunter, "Partial discharge monitoring of power transformers using UHF sensors. Part I: sensors and signal interpretation," IEEE Electrical Insulation Magazine, vol. 21, no. 2, pp. 5-14, 2005.

[8] E. Gulski, "Discharge pattern recognition in high voltage equipment," in International Conference on Partial Discharge, 1993, pp. 36-38.

[9] N. Aziz, M. Judd, and V. Catterson, "Identifying prognostic indicators for electrical treeing in solid insulation through PD analysis," in IEEE International Conference on Solid Dielectrics (ICSD), 2013, pp. 152-155.

[10] L. Li, J. Tang, and Y. Liu, "Partial discharge recognition in gas insulated switchgear based on multi-information fusion," IEEE Transactions on Dielectrics and Electrical Insulation, vol. 22, no. 2, pp. 1080-1087, 2015.

[11] Suwarno, "Partial discharges in high voltage insulations: Mechanism, patterns and diagnosis," in IEEE International Conference on Electrical Engineering and Computer Science (ICEECS), 2014, pp. 369-375.

[12] R. Patsch and F. Berton, "Pulse Sequence Analysis-a diagnostic tool based on the physics behind partial discharges," Journal of Physics D: Applied Physics, vol. 35, no. 1, p. 25, 2002.

[13] I. Bruce, B. Stewart, and C. Zhou, "An examination of the effectiveness of Pulse Sequence Analysis on PD data from artificial void defects in PET," in 47th International Universities Power Engineering Conference (UPEC), 2012, pp. 1-6.

[14] H. R. Mirzaei, A. Akbari, M. Allahbakhshi, and M. Kharezi, "New attempts in automated partial discharge identification using pulse sequence analysis," in IEEE 9th International Conference on the Properties and Applications of Dielectric Materials (ICPADM), 2009, pp. 493-496.

[15] N. Chalashkanov, S. Dodd, L. Dissado, and J. Fothergill, "Pulse sequence analysis on PD data from electrical trees in flexible epoxy resins," in Annual Report: Conference on Electrical Insulation and Dielectric Phenomena (CEIDP), 2011, pp. 776-779.

[16] H. Kaneiwa, Y. Suzuoki, and T. Mizutani, "Characteristics of partial discharges in artificial simulated tree channels during tree propagation," IEEE Transactions on Dielectrics and Electrical Insulation, vol. 8, no. 1, pp. 72-77, 2001.

[17] L. A. Dissado and J. C. Fothergill, Electrical Degradation and Breakdown in Polymers. Peter Peregrinus Ltd, 1992.

[18] A. Cavallini, M. Conti, G. Montanari, C. Arlotti, and A. Contin, "PD inference for the early detection of electrical treeing in insulation systems," IEEE Transactions on Dielectrics and Electrical Insulation, vol. 11, no. 4, pp. 724-735, 2004.

[19] R. Vogelsang, B. Fruth, T. Farr, and K. Fröhlich, "Detection of electrical tree propagation by partial discharge measurements,"
European Transactions on Electrical Power, vol. 15, no. 3, pp. 271-284, 2005.

[20] J. Champion and S. Dodd, "Systematic and reproducible partial discharge patterns during electrical tree growth in an epoxy resin," Journal of Physics D: Applied Physics, vol. 29, no. 3, p. 862, 1996.

[21] J. B. Coble, "Merging data sources to predict remaining useful lifean automated method to identify prognostic parameters," 2010.

[22] R. Liao, L. Yang, J. Li, and S. Grzybowski, "Aging condition assessment of transformer oil-paper insulation model based on partial discharge analysis," IEEE Transactions on Dielectrics and Electrical Insulation, vol. 18, no. 1, pp. 303-311, 2011.

[23] W. Yan, "On reducing feature dimensionality for partial discharge diagnosis applications," in IEEE Conference on Prognostics and System Health Management (PHM), 2012, pp. 1-7.

[24] A. Rizzi, F. M. F. Mascioli, F. Baldini, C. Mazzetti, and R. Bartnikas, "Genetic optimization of a PD diagnostic system for cable accessories," IEEE Transactions on Power Delivery, vol. 24, no. 3, pp. 1728-1738, 2009.

[25] R. Sharkawy, K. Ibrahim, M. Salama, and R. Bartnikas, "Particle swarm optimization feature selection for the classification of conducting particles in transformer oil," IEEE Transactions on Dielectrics and Electrical Insulation, vol. 18, no. 6, pp. 1897-1907, 2011.

[26] S. Bahadoorsingh and S. Rowland, "Investigating the impact of harmonics on the breakdown of epoxy resin through electrical tree growth," IEEE Transactions on Dielectrics and Electrical Insulation, vol. 17, no. 5, pp. 1576-1584, 2010.

[27] N. Aziz, V. Catterson, M. Judd, S. Rowland, and S. Bahadoorsingh, "Prognostic modeling for electrical treeing in solid insulation using pulse sequence analysis," in IEEE Conference on Electrical Insulation and Dielectric Phenomena (CEIDP), 2014, pp. 373-376.

[28] N. Aziz, V. Catterson, S. Rowland, and S. Bahadoorsingh, "Effect of harmonics on Pulse Sequence Analysis plots from electrical trees," in IEEE Conference on Electrical Insulation and Dielectric Phenomena (CEIDP), 2014, pp. 377-380.

[29] S. Bahadoorsingh and S. Rowland, "Investigating the influence of the lubricant coating on hypodermic needles on electrical tree characteristics in epoxy resin," IEEE Transactions on Dielectrics and Electrical Insulation, vol. 17, no. 3, pp. 701-708, 2010.

[30] G. Montanari, "Notes on theoretical and practical aspects of polymeric insulation aging," IEEE Electrical Insulation Magazine, vol. 4, no. 29, 2013.

[31] J. I. Aizpurua and V. M. Catterson, "Towards a Methodology for Design of Prognostic Systems," in Annual Conference of the Prognostics and Health Management Society, Coronado, California, USA, 2015.

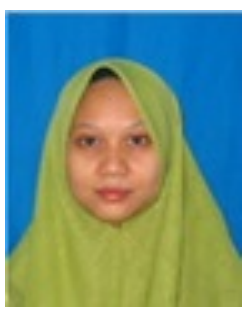

Nur Hakimah Ab Aziz (S'13) was born in Melaka, Malaysia. She completed the B.Eng. in Electrical Engineering at Universiti Teknologi Malaysia in 2003 and M.Eng. at University of South Australia in 2006. She is a lecturer in Universiti Teknikal Malaysia Melaka and currently pursuing her Ph.D. degree at the University of Strathclyde. Her research interests include condition monitoring, diagnostics and prognostics and dielectric ageing.

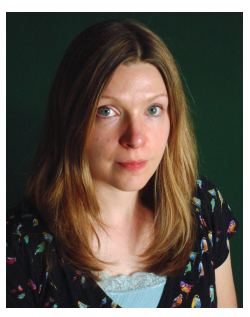

Victoria M. Catterson (M'03, SM'12) received the B.Eng. (Hons.) and Ph.D. degrees from the University of Strathclyde, Glasgow, U.K., in 2003 and 2007, respectively. She is a lecturer within the Institute for Energy and Environment at the University of Strathclyde. Her research interests include intelligent systems for applications in the power industry. She is the Chair of the IEEE Dielectrics and Electrical Insulation Society Technical Committee on Smart Grids. 


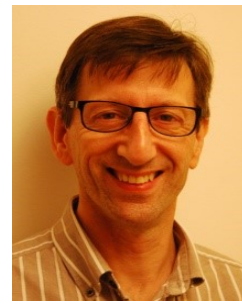

Prof Simon M Rowland (F'14) was born in London, England. He completed the B.Sc. degree in physics at The University of East Anglia, and the $\mathrm{PhD}$ degree at London University, UK. He has worked for many years on dielectrics and their applications and has also been Technical Director within multinational companies. He joined The School of Electrical and Electronic Engineering in The University of Manchester in 2003, and was appointed Professor of Electrical Materials in 2009, and Head of School in 2015. Prof. Rowland was President of the IEEE Dielectric and Electrical Insulation Society from 2011-12.

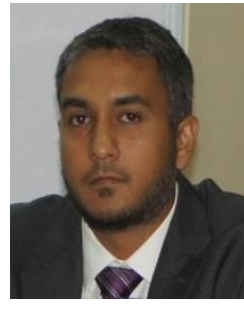

Sanjay Bahadoorsingh (M'03, SM'16) was born in Port of Spain, Trinidad. He completed the B.Sc. degree from The University of The West Indies in 2003 and the M.Sc. degree from UMIST in 2005. In 2009, he completed the Ph.D. degree at The University of Manchester. He is currently a lecturer in the Energy Systems Group at The University of the West Indies. His areas of research are power systems operation and planning, renewable energy integration, asset management and dielectric ageing. 